\title{
A move ahead on headache
}

\section{The genetics of inherited migraines is proving more tractable than might have been expected given the clinical heterogeneity of the malady.}

IT is surely a sign of the times that clinical geneticists are willing to take on such ephemeral conditions as headaches and try and pin them down genetically. Who in their right mind would choose to tackle a condition that for a long time has defied definition, not to mention diagnosis or genetic analysis? Yet this is precisely what a French group, led by Elisabeth Tournier-Lasserve (Enfants Malades, Paris), has done and with some success. In the September issue of Nature Genetics, Joutel et al. ${ }^{1}$ describe a linkage study that shows that a particular type of migraine maps to a 30-centimorgan region of chromosome 19.

With any other condition such preliminary linkage would only rarely warrant publication before the region of interest had been narrowed down a little further, but one look at what is involved in the genetics of headaches is enough to see the major advance that this represents. It is also enough to appreciate that the French group is not dealing with the morningafter symptoms of a good night out, but rather with a specific and very rare type of inherited migraine which has a reasonably clear autosomal dominant mode of inheritance and some very serious symptoms other than a sore head.

In general, migraine is a common and distressing type of headache for which there is no known aetiology. It affects more women (estimated at 18 per cent) than men ( 6 per cent) and shows broad clinical heterogeneity. In the absence of laboratory-based diagnostic tests it has, until recently, been difficult to establish whether two neurologists might be experiencing the same headache, never mind what is going on inside the head of, for example, a five-year-old victim of migraine. To address this problem of diagnostic variability, the International Headache Society proposed a new classification system that would allow all types of headache, including migraine, to be defined using a common set of rules.

Also in this month's Nature Genetics: two reports show Charcot-Marie-Tooth neuropathy type $\mathrm{IB}$ is associated with mutations in the myelin protein Po; hemizygosity of the elastin gene causes Williams syndrome; point mutations in c-myc are frequent in Burkitt's lymphoma and mouse plasmacytomas; and IGF2 is imprinted in mouse but not in humans.
Migraine is thus generally defined as a severe and pulsating head pain that lasts for several hours and is associated with nausea and perhaps vomiting. Approximately ten per cent of migraines are preceded by what neurologists refer to as an aura, and it is into this subgroup that familial hemiplegic migraine - the subject of the new paper by Joutel and colleagues - falls.

In familial hemiplegic migraine (FHM), the aura consists of hemiplegia (complete paralysis of half of the body) or hemiparesis (partial paralysis). This motor disturbance phase, which can be brought on by trauma to the head, typically lasts for about an hour. Although there can be some delay between the end of the aura and the start of the headache, the severe migraine-type headache may follow immediately.

For some forty or fifty years it has been clear that genetic factors are at play in migraine. In light of the efficiency with which linkage studies can be carried out these days, it is perhaps a little surprising that no one had considered collecting specimens from affected families before with a view to linkage analysis. But a look at the complexity of headache diagnosis explains this reluctance. In fact, the current discovery came about in part through a fortunate coincidence.

During investigations of a much more severe and what has, until now, been considered a completely separate disorder, cerebral autosomal dominant arteriopathy with subcortical infarcts and leukoencephalopathy (CADASIL), it was noticed that some of these patients also suffered recurrent attacks of severe headache. With serendipity in full flight, it was postulated that, although clinically distinct, the two disorders might be allelic.

CADASIL was previously mapped to chromosome 19 (by the same group ${ }^{2}$ ) and thus close markers were available. Tournier-Lasserve and colleagues chose two well defined FHM families (made up of more than fifty individuals, thirty of whom were affected according to the criteria of the International Headache Society) and performed linkage analysis using the CADASIL-linked markers. To everyone's surprise and delight, they turned up a combined positive lod score of over 8 in favour of linkage to chromosome 19. Multipoint analysis suggested that both CADASIL and FHM mapped to a 30 -centimorgan region, and although preliminary ordering of some half-dozen markers and the two disease loci suggests that the two conditions most probably map to different intervals, it is still possible that they could be due to defects in a common gene. Any suggestion that CADASIL and FHM are associated comes as a surprise to neurologists, who have always considered the two conditions to be totally independent of one another. This work has therefore forced a rethink of the possible aetiology of both CADASIL and inherited migraine. More families are being recruited into the study, which will now concentrate on narrowing the chromosome-19 region of interest and defining the nature of the link between CADASIL and FHM.

The French group reported their exciting news at the sixth congress of the International Headache Society, held in Paris this summer. The congress organizer, Marie-Germaine Bousser, a prominent figure in the society (and a senior author on the Joutel et al. paper) points out that previous meetings have been dominated by discussion of drug-based treatments for migraine. For the first time, the congress devoted a full session to the genetics of migraine, with six independent groups presenting results of family-based studies. This reflects a growing awareness that many types of severe headache may show multifactorial inheritance. TournierLasserve and her colleagues are therefore interested in examining other types of migraine headache that have similar although distinct characteristics for chromosome-19 associations. Other categories of headache, such as the very common and clinically even more diverse tensiontype headaches, are thought to be caused mainly by environmental factors and to have little or no familial association.

As for help for those of us who suffer what we consider to be migraine, but which is in truth only a very trivial and temporary headache, we can take comfort in the knowledge that we have available to us the same treatment that is relied upon by the less fortunate sufferers of FHM: an aspirin.

Adrian J. Ivinson

Adrian J. Ivinson is Assistant Editor of Nature Genetics.

\footnotetext{
1. Joutel, A. et al. Nature Genet. 5, 40-45 (1993).

2. Tournier-Lasserve, E. et al. Nature Genet. 3, 256-259 (1993).
} 